\title{
LARGE-SCALE STRUCTURE
}

\section{Topography of darkness}

Dark matter and dark energy regulate the way that large-scale structure forms and evolves in the Universe. The latest observations of the cosmic microwave background by the Planck satellite indicate that almost $84 \%$ of the total matter in the Universe is dark and therefore cannot be observed directly. Rather, its properties and distribution can only be inferred through its - predominantly gravitational - interaction with luminous matter. Priyamvada Natarajan and collaborators are now putting the $\Lambda$ cold dark matter ( $\Lambda$ CDM) cosmological model to the test by scrutinizing the way that dark matter congregates compared to our theoretical expectations (Mon. Not. R. Astron. Soc., in the press; preprint at https://arxiv.org/abs/1702.04348).

Gravitational lensing is one of the most secure ways to weigh the total matter content of clusters of galaxies since both visible and dark matter contribute to the total gravitational potential of the 'lens'. Natarajan et al. focused their study on three galaxy clusters observed by the Hubble Space Telescope. The high

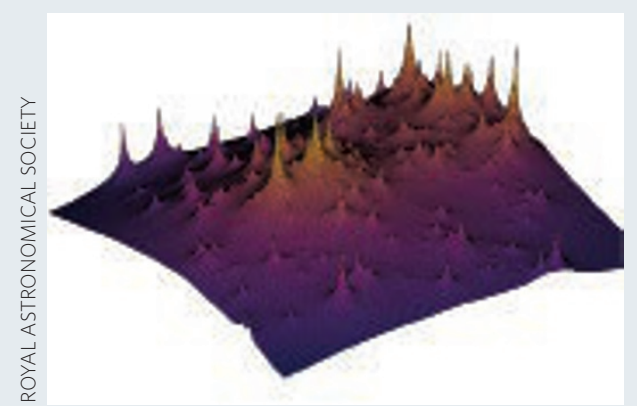

sensitivity afforded by Hubble allowed the authors to build accurate models of the total lensing mass in each cluster and to identify substructures in its distribution. These subhaloes can be visualized as spikes in the two-dimensional map of the mass density (pictured for one of the clusters). The amplitude, shape and radial distribution of each cluster's subhalo mass function - the number of subhaloes per subhalo mass bin - were then contrasted to the expectations from analytical models and the Illustris $\Lambda$ CDM cosmological simulation.

Reassuringly for the $\Lambda$ CDM paradigm, both the amplitude and the shape of all three subhalo mass functions are found to match theoretical expectations very well, especially after correcting for confounding factors such as projection effects and, perhaps more importantly, biases due to cosmic variance. This result indicates that there is no mass 'missing' at cluster scales, in contradiction to previous claims based on studies of the abundance and distribution of satellite galaxies around the Milky Way.

Natarajan et al. however do find a discrepancy between the observed and expected radial distribution of the dark matter. The authors interpret this mismatch as an indication that the observed clusters are dynamically unrelaxed, caught during a short-lived phase of their evolution marked by interactions and mergers between the cluster members. Such a phase cannot be accurately 'captured' by current-generation simulations as it would require a much denser temporal sampling and a more realistic treatment of tidal stripping and dynamical friction.

MARIOS KAROUZOS 